\section{Ciclamato de sódio e rim fetal}

\section{Sodium cyclamate and fetal kidney}

\begin{abstract}
Cyclamate is used as an artificial noncaloric sweetener in a variety of foods and beverages, being 30 times as sweet as sugar without the bitter after-taste of saccharin. It is present in the formula of products such as sodium and calcium cyclamates and cyclamic acid. Cyclamate and cyclohexylamine, its principal metabolite, can cross the human placenta exposing the fetus. It has been demonstrated in rats that the kidney may be adversely affected by high doses of cyclamate. Studies on the effects of sodium cyclamate in the human species are necessary, because in addition to replacing saccharose - harmful in individuals with diabetes or patients in whom weight reduction and control are essential for health - it does not cause dental caries.
\end{abstract}

Key words Sodium cyclamate, Kidney, Fetal health
José Germano Ferraz de Arruda 1

Alex Tadeu Martins 2

Reinaldo Azoubel 3

\begin{abstract}
1 Departamento de Especialidades Cirúrgicas. Faculdade de Medicina de São José do Rio Preto. Av. Brigadeiro Faria Lima, 5416. São José do Rio Preto, SP, Brasil. CEP: 15.090-000. E-mail: urologiarruda@ig.com.br

2,3 Programa de Pós-Graduação em Ciências da Saúde. Faculdade de Medicina de São José do Rio Preto, SP.
\end{abstract}

Resumo O ciclamato é usado como adoçante artificial não calórico em diversos alimentos e bebidas, sendo 30 vezes mais doce que a sacarose sem o sabor amargo da sacarina. Aparece na composição dos produtos como ciclamato de sódio, ciclamato de cálcio e ácido ciclâmico. O ciclamato e a ciclohexilamina, seu principal metabólito, atravessam a barreira placentária em humanos e desse modo podem ser expostos ao feto. $O$ rim de ratos pode ser afetado por elevadas doses de ciclamato de sódio. Estudos sobre efeitos do ciclamato de sódio na espécie humana são necessários, pois, além de poder substituir a sacarose - prejudicial em casos de diabetes ou quando o controle e a redução do peso corporal são essenciais para a saúde dos pacientes - não propicia desenvolvimento de cárie dentária.

Palavras-chave Ciclamato de sódio, Rim, Saúde fetal. 
O ciclamato, sal do ácido N-ciclo-hexil-sulfâmico (CHS), é usado como adoçante artificial não calórico em diversos alimentos e bebidas, e na indústria farmacêutica. Aparece na composição dos produtos em três diferentes formas: ciclamato de sódio $\left(\mathrm{C}_{6} \mathrm{H}_{11} \mathrm{NHSO}_{3} \mathrm{Na}\right)$, ciclamato de cálcio $\left(\mathrm{C}_{12} \mathrm{H}_{24} \mathrm{~N}_{2} \mathrm{~S}_{2} \mathrm{O}_{6} \mathrm{Ca}\right)$ e ácido ciclâmico $\left(\mathrm{C}_{6} \mathrm{H}_{13} \mathrm{NO}_{3} \mathrm{~S}\right) .{ }^{1}$ É inodoro, solúvel em água, álcool e propilenoglicol;2 é mais estável que o aspartame e a sacarina, podendo por isso ser levado a altas e a baixas temperaturas. 3

A descoberta do ciclamato ocorreu em 1937 por Michael Sveda, aluno de graduação da University of Illinois, Estados Unidos, que casualmente descobriu seu sabor adocicado, 30 vezes mais doce que a sacarose, sem o sabor amargo da sacarina. ${ }^{4}$ Essa substância foi inicialmente comercializada (em 1949) como adoçante artificial para diabéticos. Em 1959, o Food and Drug Administration (FDA), adicionou o ciclamato à lista das substâncias reconhecidas como seguras. 5

A partir da mistura de ciclamato com sacarina na proporção 10:1, houve aumento do consumo de adoçantes artificiais nos Estados Unidos na década de 60. Em 1968, foram produzidas $7.718 \mathrm{t}$ de ciclamato, sendo $69 \%$ utilizado em bebidas, $19 \%$ em adoçantes de mesa, $6 \%$ em alimentos, $4 \%$ em itens não alimentares e $2 \%$ exportado. 6 Segundo Burbank e Fraumeni,7 em 1969 foram consumidos, nesse país, 8.943 t de ciclamato.

Em meados da década de 60, Kojima e Ichibagase 8 constataram que o ciclamato de sódio não era eliminado de forma invariável, mas poderia ser metabolizado como ciclohexalamina. Em 1969, o ciclamato associado à sacarina na proporção 10:1 foi interpretado pelo FDA como indutor de câncer de bexiga em ratos.9,10 A partir desses estudos, seu uso foi proibido nos Estados Unidos após o US Department of Health, Education and Welfare concluir que o ciclamato não apresentava qualquer valor no tratamento da obesidade ou diabetes. ${ }^{6}$ Apesar disso, o World Health Organization's Joint Expert Committee on Food Additives aprovou o uso do ciclamato de sódio em 1977 como adoçante em alimentos e bebidas em mais de 40 países, 11 incluindo Brasil, Alemanha, Finlândia, Paquistão, África do Sul e Suíça. ${ }^{5}$

Kroes et al.,12 estudando a toxicidade a longo prazo do ciclamato, sacarina e ciclohexilamina em ratos, e seus efeitos na reprodução, constataram que nenhuma dessas substâncias apresentou efeito teratogênico ou afetou a reprodução, sendo apenas a ciclohexilamina embriotóxica.

O ciclamato, quando ingerido em grandes quantidades, produz diarréia em humanos. De acordo com Egeberg et al., 6 isso é aparentemente o resultado de um efeito osmótico, pois não há evidência de que essa substância agrave doenças gastrointestinais orgânicas.

Em seres humanos e em várias espécies animais o ciclamato não é absorvido completamente no intestino. ${ }^{13}$ Quando absorvido é, rapidamente excretado na urina sem considerável acúmulo no sangue ou tecidos. 14 A maior parte do ciclamato não absorvido é normalmente eliminada nas fezes, mas uma quantidade variável é convertida para ciclohexilamina por microorganismos que habitam o colon e o cecum. 15

A conversão do ciclamato para ciclohexilamina em humanos é heterogênea, sendo que aproximadamente $76 \%$ dos usuários regulares convertem menos do que $0,1 \%$ de uma dose ingerida de ciclamato, cerca de oito a $10 \%$ convertem $1 \%$ ou mais e $4 \%$ convertem $20 \%$ ou mais. 5 Collings, 16 investigando o metabolismo do ciclamato de sódio em humanos, constatou que com o aumento da dose dessa substância a quantidade de ciclohexilamina excretada também aumenta. Entretanto, segundo o autor, a porcentagem de conversão diminui com o aumento da dose de ciclamato de sódio.

O principal metabólito do ciclamato, a ciclohexilamina, é rapidamente absorvido e excretado pelos rins, existindo pouca excreção fecal. As taxas de excreção urinária de ciclamato e ciclohexilamina indicam que pouco permanece nos tecidos ou fluidos corpóreos após sua administração prolongada e em doses elevadas. 13 Além disso, ambos, ciclamato e ciclohexilamina, podem ser transportados pela barreira placentária e desse modo podem ser expostos ao feto. 17,18 De acordo com Pitkin et al.,19 os ciclamatos atravessam a placenta e, na circulação fetal, alcançam $1 / 4$ da concentração materna, sendo encontrados, de preferência, no fígado e nos rins.

Vários produtos do metabolismo da ciclohexilamina foram identificados em humanos, como o ciclohexanol, a ciclohexanona e a trans-ciclohexanona-1,2-diol. 20 No entanto, a inexistencia de qualquer diferença entre as rotas de administração com relação à extensão do metabolismo da ciclohexilamina implica que os tecidos, mais do que a flora intestinal, constituem o principal local para conversão desse metabólito. 21

Diversos estudos foram realizados em animais de laboratório visando verificar toxicidade do ciclamato de sódio ou da sua mistura com a sacarina.22,23 Os resultados revelaram poucos efeitos fisiopatológicos associados com a administração dessa substância, mesmo em doses elevadas. Considerando a grande quantidade de trabalhos publicados na década de 60 sobre toxicidade do ciclamato, efeitos 
adversos foram relatados e atraíram a atenção da comunidade científica, sendo que muitas dessas contribuições foram consideradas achados isolados. 11

Analisando aspectos toxicológicos do ciclamato de sódio e da ciclohexilamina, Bopp et al. ${ }^{11}$ apresentam revisão sobre efeitos dessas substâncias em órgãos e sistemas como o fígado (sem alteração histológica), o rim (nefrocalcinose, nefrite e nefrose), o trato gastrointestinal (diarréia), o coração (calcificação no miocárdio e esclerose de valvas), o sangue (anemia e plaquetopenia), a tireóide (elevação da quantidade de iodo ligado à proteína), a adrenal (aumento do peso), o pâncreas (aumento da quantidade de células alfa-pancreáticas) e o sistema reprodutor (oligospermia) em animais.

Takayama et al.,24 investigando a toxicidade e carcinogenicidade a longo prazo em macacos alimentados com $100 \mathrm{mg} / \mathrm{kg}$ e $500 \mathrm{mg} / \mathrm{kg}$ de ciclamato, cinco vezes por semana, durante 24 anos, constataram espermatogênese irregular e casos esporádicos de diferentes malignidades. Segundo os referidos autores, esses achados não fornecem evidência clara de efeito tóxico ou carcinogênico do ciclamato de sódio em macacos.

Segundo alguns autores, o rim de ratos pode ser afetado por elevadas doses de ciclamato de sódio. Em estudos crônicos tem sido relatado leve aumento na incidência de nefrite e de nefrose. Entretanto, tais alterações também foram comuns em ratos controle. 25 A alteração mais freqüente atribuída ao ciclamato envolve calcificação nos rins, seguida, em alguns casos, por hiperplasia do epitélio renal. 26

Friedman et al.,25 investigando ratos submetidos à dietas contendo $0,4 \%, 2 \%$ ou $10 \%$ de ciclamato de sódio ou cálcio durante 88 a 101 semanas, registraram nefrocalcinose em 5\% dos ratos controle e 47 a $49 \%$ daqueles que receberam ciclamato. A incidência de nefrocalcinose estava relacionada à dose, pois na maioria dos animais com tal alteração tinha sido fornecido $10 \%$ de ciclamato. Entretanto foi similar com os sais de sódio e de cálcio.

Estudos clínicos têm indicado que a administração de ciclamato de sódio em doses de dois a $10 \mathrm{~g} /$ dia não afeta a função renal em humanos. 27 Zöllner e Pieper, 28 estudando pacientes com doenças crônicas renais e hepáticas que receberam doses diárias de ciclamato de sódio (dois ou cinco g) por mais de três anos, verificaram, por meio de monitorização de variáveis bioquímicas e resultados de uroanálise, que não havia qualquer efeito negativo na função renal desses pacientes.

Becker e Gibson, ${ }^{29}$ avaliando a teratogenicidade da ciclohexilamina em ratos, registraram dois casos de hidronefrose num total de 84 fetos. Segundo os autores, esse achado parece não estar relacionado com as doses ingeridas (61 e $122 \mathrm{mg} / \mathrm{kg}$ ).

Atualmente, existem no Brasil diversos adoçantes de mesa à base de ciclamato e sacarina, sendo que os mais vendidos possuem a proporção de duas partes de ciclamato para uma de sacarina. 30 Assunção et al.,31 avaliando o conhecimento e a quantidade de adoçante consumida diariamente por 36 indivíduos diabéticos, constataram que $92 \%$ deles consomem adoçantes artificiais não calóricos à base de ciclamato e sacarina em quantidades inferiores à ingestão diária aceitável (IDA). De acordo com a Food and Agriculture Organization (FAO) e o World Health Organization (WHO) Expert Committee on Food Additives, a IDA do ciclamato corresponde a $50 \mathrm{mg} / \mathrm{kg}$ de peso corporal. ${ }^{10}$

Investigações sobre efeitos de substâncias como o ciclamato de sódio na espécie humana são necessárias, pois além de substituir a sacarose, prejudicial em casos de diabetes ou no controle e redução do peso corporal, não propicia desenvolvimento de cárie dentária. Entretanto, vale ressaltar que pesquisas com animais de laboratório possibilitam obter, em pouco tempo e em condições controladas, informações a respeito do potencial tóxico de substâncias químicas sobre o organismo em desenvolvimento. Além disso, a maior parte das publicações de pesquisas sobre efeitos do ciclamato de sódio ocorreram nas décadas de 60 e 70, reduzindo-se posteriormente, em particular, devido à proibição do uso dessa substância pelo FDA dos Estados Unidos, em 1969.6

Estudos sobre o efeito do ciclamato de sódio no rim e no fígado de fetos de ratas estão sendo realizados na Faculdade de Medicina de São José do Rio Preto, SP, no período de maior teratogenicidade da espécie. Resultados preliminares têm indicado nefrotoxicidade, retardo no desenvolvimento fetal e índice de maturação placentária reduzido. 


\section{Referências}

1. Cattanach BM. The mutagenicity of cyclamates and their metabolites. Mutat Res 1976; 39: 1-28.

2. Sain OL, Berman JM. Efectos adversos de edulcorantes en pediatria sacarina y ciclamato. Arch Arg Pediatr 1984; 82: 209-11.

3. Barlattani M. Rassegne sintetiche di terapia. II problema dei ciclamati. Rass Clin Ter Sci Affini 1970; 52: 565-0.

4. Audreith LF, Sveda M. Preparation and properties of some N-substituted sulphamic acids. J Org Chem 1944; 9: 89101.

5. Ahmed FE, Thomas DB. Assessment of the carcinogenicity of the nonnutritive sweetner cyclamate. Crit Rev Toxicol 1992; 22: 81-118.

6. Egeberg RO, Steinfeld JL, Frantz I, Griffith GC, Knowles JR RH, Rosenow E, Sebrell H, Van Itallie T. Report to the secretary of HEW from the Medical Advisory Group on cyclamates. JAMA 1970; 211: 1358-61.

7. Burbank F, Fraumeni JFJr. Synthetic sweetener consumption and bladder cancer trends in the United States. Nature 1970; 227: 296-7.

8. Kojima S, Ichibagase H. Studies on synthetic sweetening agents. VIII. Cyclohexylamine, a metabolite of sodium cyclamate. Chem Pharm Bull 1966; 14: 971-4.

9. Price JM, Biava CG, Oser BL, Vogin EE, Steinfeld J, Ley HL. Bladder tumors in rats fed cyclohexylamine or high doses of a mixture of cyclamate and saccharin. Science 1970; 167: 1131-2

10. Oser BL, Carson S, Cox GE, Vogin EE, Sternberg SS. Chronic toxicity study of cyclamate: saccharin $(10: 1)$ in rats. Toxicology 1975; 4: 315-30.

11. Bopp BA, Sonders RC, Kesterson JW. Toxicological aspects of cyclamate and cyclohexamine. Crit Rev Toxicol 1986; 16: 213-306.

12. Kroes R, Peters PWJ, Berkvens JM, Verschuuren HG, De Vries TH, Van Esch GJ. Long-term toxicity and reproduction study (including a teratogenicity study) with cyclamate, saccharin and cyclohexylamine. Toxicology 1977; 8: 285-300.

13. Dick CE, Schniepp ML, Sonders RC, Wiegand RG. Cyclamate and cyclohexylamine: lack of effect on the chromosomes of man and rats in vivo. Mutat Res 1974; 26: 199203.

14. Collings AJ. Metabolism of cyclamate and its conversion to cyclohexylamine. Diabetes Care 1989; 12: 50-5

15. Renwick AG, Williams RT. The metabolites of cyclohexylamine in man and certain animals. Biochem J 1972; 129: $857-67$

16. Collings AJ. The metabolism of sodium cyclamate. In: Birch GG, editor. Sweetness and sweeteners. London: Applied Science; 1971. p. 51-68.
17. Pitkin RM, Reynolds WA, Filer LJ. Cyclamate and cyclohexylamine: transfer across the hemochorial placenta. Proc Soc Exp Biol Med 1969; 132: 993-5.

18. Schechter PJ, Roth LJ. Whole-body autoradiography of $14 \mathrm{C}$ sodium cyclamate in pregnant and fetal rats. Toxicol Appl Pharmacol 1971; 20: 130-3.

19. Pitkin RM, Reynolds WA, Filer LJ. Placental transmission and fetal distribution of cyclamate in early human pregnancy. Am J Obstet Gynecol 1970; 108: 1043-50.

20. Kojima S, Ichibagase H. Studies on synthetic sweetening agents. XII. The binding of sodium cyclamate with bovine serum albumin. Chem Pharm Bull 1968; 16: 1619-22.

21. Roberts A, Renwick AG. The metabolism of 14C-cyclohexylamine in mice and two strains of rat. Xenobiotica 1985; 15: 477-83.

22. Dalderup LM, Visser W. Influence of extra sucrose, fats, protein and of cyclamate in the daily food on the lifespan of rats. Experientia 1971; 15: 519-21.

23. Ferrando R, Huchet B. Etude de l'activité éventuelle du cyclamate de soude sur le rat au cours de trois générations. Bull Acad Natl Med 1968; 153: 36-41.

24. Takayama S, Renwick AG, Johansson SL, Thorgicirsson UP, Tsusumi M, Dalgard DW, Sieber SM. Long-term toxicity and carcinogenicity study of cyclamate in nonhuman. Toxicol Sci 2000; 53: 33-9.

25. Friedman L, Richardson HL, Richardson ME, Lethco EJ, Wallace WC, Sauro FM. Toxic response of rats to cyclamates in chow and semisynthetic diets. J Natl Cancer Inst 1972; 49: 751-64.

26. Oser BL, Carson S, Vogin EE. Growth and reproduction studies with cyclamate-saccharin (10:1) in rats. Toxicol Appl Pharmacol 1968; 12: 290-1.

27. Bernryma GH, Hazel GR, Taylor JD, Sanders PG, Weinberg MS. A case for safety of cyclamate and cyclamatesaccharin combinations. Am J Clin Nutr 1968; 21: 67387.

28. Zöllner VN, Pieper M. Concluding report of a 3-year clinical study on cyclamate. Arzneimittelforschung 1971; 21: 431-2.

29. Becker BA, Gibson JE. Teratogenicity of cyclohexylamine in mammals. Toxicol Appl Pharmacol 1970; 17: 551-2.

30. Cardello HMAB, Silva MAAP, Damásio MH. Análise descritiva quantitativa de edulcorantes em diferentes concentrações. Ci Tecnol Aliment 2000; 20: 318-28.

31. Assunção MCF, Andersson GB, Cavalcanti ZCH. Uso de adoçantes alternativos pelos diabéticos. J Bras Med [JBM] 1994; 67: 62-9.

Recebido em 6 de janeiro de 2003

Reapresentado em 3 de abril de 2003

Aprovado em 28 de abril de 2003 